\title{
Pneumothorax « radio-occulte »
}

\section{Occult pneumothorax}

\author{
K. Habchi • T. Schmutz • N. Guler • F. Braun \\ (C) SFMU et Springer-Verlag France 2013
}

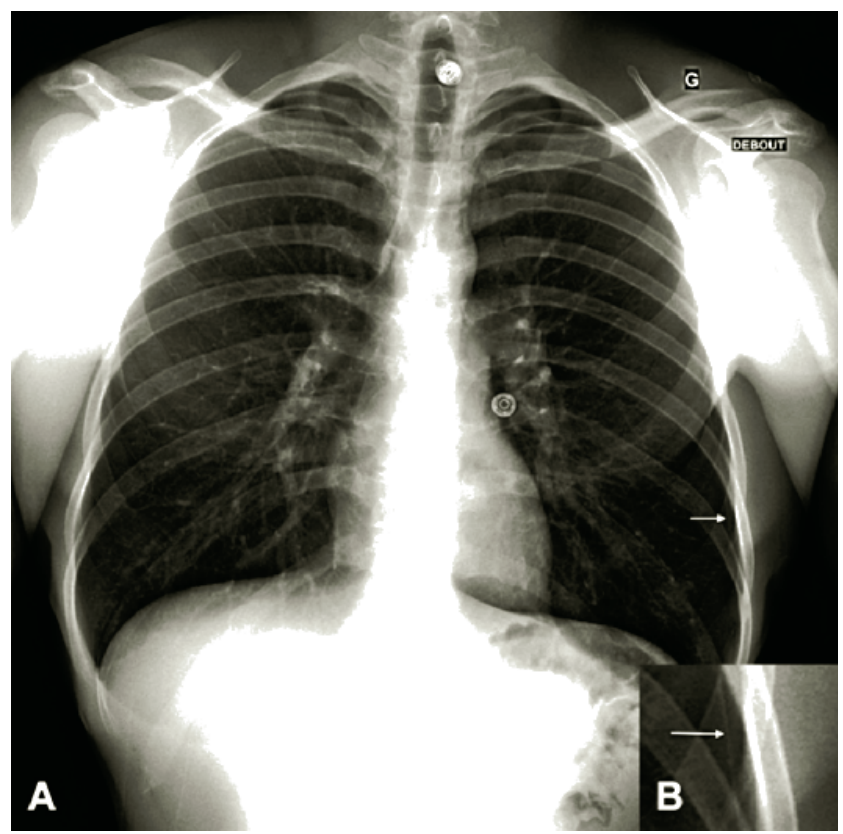

Fig. 1 Radiographie thoracique de face : le cliché (A) paraît normal à la première lecture. Une analyse plus fine permet de déceler le décollement (flèche blanche) au niveau du lobe inférieur gauche (agrandissement B)

Un homme consulte aux urgences pour douleur thoracique. À l'examen clinique, le murmure vésiculaire est diminué à gauche. Sur la radiographie thoracique de face, seule la visibilité de la plèvre viscérale au niveau du lobe inférieur gauche permet d'évoquer la présence d'un pneumothorax (Fig. 1). Un scanner thoracique réalisé sans délai devant la mauvaise tolérance met en évidence un volumineux épanchement gazeux antérieur gauche (Fig. 2). Un pneumothorax peut ne pas être visible sur une radiographie thoracique

K. Habchi $\cdot$ T. Schmutz $(\square) \cdot$ N. Guler $\cdot$ F. Braun Service des urgences, Samu-Smur, CHR Metz-Thionville, 1, allée du château, F-57085 Ars-Laquenexy, France e-mail : t.schmutz@chr-metz-thionville.fr

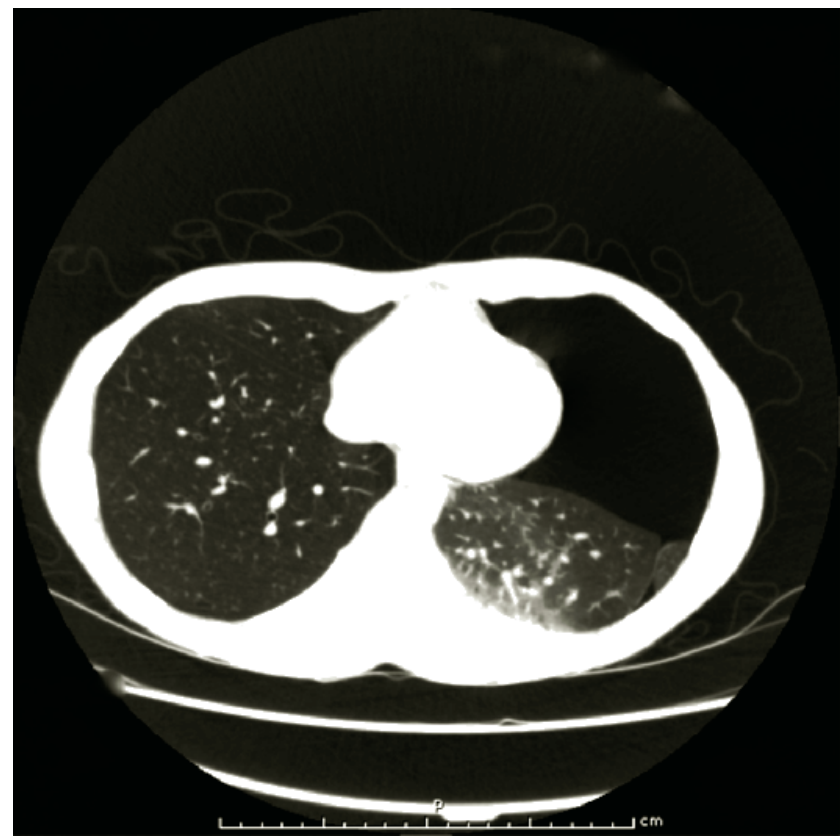

Fig. 2 Scanner thoracique sans injection de produit de contraste

(pneumothorax « radio-occulte »). Certains signes radiologiques (aplatissement du diaphragme, déplacement médiastinal, poumon « hyperclair », visibilité de la plèvre) doivent alerter. Le cliché standard en expiration profonde peut rendre visible le décollement, mais risque d'être mal toléré en cas de pneumothorax important. Même si l'échographie pulmonaire peut affirmer le diagnostic avec une très forte sensibilité [1], le scanner thoracique reste l'examen de référence. Il pose le diagnostic et évalue l'abondance de l'épanchement avec certitude [2].

\section{Références}

1. Lichtenstein D, Mezière G, Biderman P, et al (2000) The "lung point": an ultrasound sign specific to pneumothorax. Intensive Care Med 26:1434-40

2. Omar HR, Abdelmalak H, Mangar D, et al (2010) Occult pneumothorax, revisited. J Trauma Manag Outcomes 4:12 